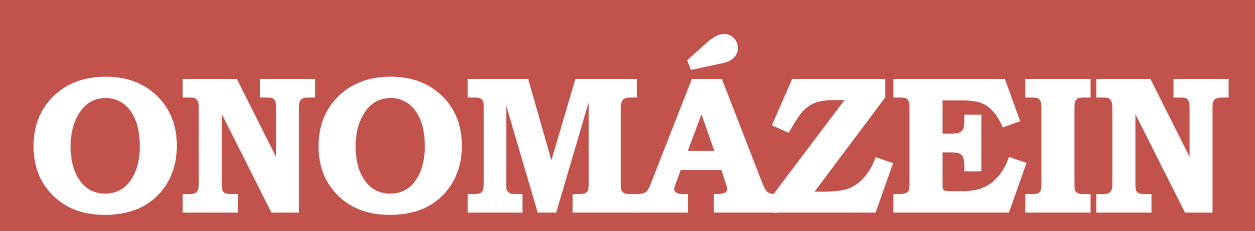

Revista de lingüística, filología y traducción
PONTIFICIA UNIVERSIDAD CATÓLICA DE CHILE FACULTAD DE LETRAS

\title{
Sobre la (hiper)extranjerización de configuraciones grafémicas
}

About the (hyper)foreignization of graphemic configurations

\section{Yolanda Hipperdinger}

Consejo Nacional de Investigaciones Científicas y Técnicas / Universidad Nacional del Sur Argentina

\section{(c) $(\mathbb{i})$}

Yolanda Hipperdinger: Consejo Nacional de Investigaciones Científicas y Técnicas / Departamento de Humanidades, Universidad Nacional del Sur, Argentina. | Correo electrónico: yhipperdinger@uns.edu.ar 


\section{Resumen}

Nos ocupamos en este artículo de una escritura no normativa de palabras españolas que involucra convenciones exógenas, distinguiéndola de una intervención que puede considerarse similar, por cuanto genera igualmente alternantes que se separan de la norma correspondiente haciendo uso de convenciones no españolas, pero para la escritura de palabras reconocidas como importaciones de otras lenguas. Sobre datos del ámbito comercial, obtenidos en el área dialectal bonaerense del español de la Argentina, comparamos la procedencia de los recursos empleados en la escritura extranjerizadora de palabras españolas y en las configuraciones hiperextranjerizadas, verificando una disparidad que ratifica la caracterización que ensayamos para ambos tipos de intervención.

Palabras clave: configuraciones grafémicas; extranjerización; hiperextranjerización.

\section{Abstract}

We have dealt in this paper, with a non-normative writing for Spanish words which implies exogenous conventions, and we have distinguished it from an intervention which can be considered similar, because it also generates alternating forms separated from the corresponding norm by using non-Spanish conventions, but for writing of words that the speakers recognize as importations from other languages. On data from the commercial domain, obtained in the Buenos Aires dialectal area of Argentine Spanish, we have compared the origin of the resources used in the foreign writing for Spanish words and in the hyperforeign configurations, verifying a disparity that ratifies the characterization that we have tried out for both types of intervention.

Keywords: graphemic configurations; foreignization; hyperforeignization. 


\section{Introducción}

En el área dialectal bonaerense del español de la Argentina, y tal como se ha descripto también para otras variedades territoriales de español, el paisaje lingüístico urbano revela una tendencia constante en la cartelería comercial: entre los recursos lingüísticos empleados en las inscripciones, un lugar de relevancia lo ocupa la recurrencia a otras lenguas (generalmente, lenguas prestigiosas). Esa recurrencia, cuyo carácter rupturista se orienta a llamar la atención del destinatario, adopta a veces la forma del cambio de código y, típicamente, la de la importación léxica y la de una escritura no normativa de palabras españolas que involucra convenciones exógenas. En este artículo nos ocupamos de este último procedimiento, presentando resultados de la investigación que desarrollamos con el objetivo principal de caracterizar el tipo de intervención implicado y, en particular, de distinguirlo del de una intervención que puede considerarse similar, en la medida en que también genera alternantes que se separan de la norma correspondiente haciendo uso de convenciones no españolas, pero para la escritura de palabras reconocidas como importaciones de otras lenguas².

En primer lugar, analizamos para ello las características de las configuraciones grafémicas extranjerizadas de palabras españolas que se registran en las denominaciones de emprendimientos comerciales de la ciudad de Bahía Blanca, representativa del área dialectal referida3, sobre los datos que ofrece la última edición de la guía telefónica respectiva. En segundo lugar, comparamos esas configuraciones con los alternantes grafémicos hipercorrectos registrados, en la publicitación in situ de productos de locales comerciales de esa ciudad, en la escritura intencionadamente conservadora de palabras reconocidas como exógenas, entendiendo que esos alternantes constituyen igualmente manifestaciones del valor social asignado a (algunas) lenguas diferentes del español. Procuramos determinar, en especial, en las convenciones de qué lenguas se abreva en la escritura extranjerizadora de palabras españolas y en los referidos alternantes hiperextranjerizados, que evidencian el intento de los productores de ajustarse a las configuraciones de origen aun sin disponer de un conocimiento seguro respecto de ellas.

1 Por la delimitación, caracterización y denominación de esta variedad territorial, véase Fontanella de Weinberg $(1987,2000)$.

2 Si bien desde una perspectiva diacrónica, como lo ha observado Haspelmath (2009: 38), no siempre es posible identificar qué palabras no han sido importadas (en el sentido precisado por Haugen, 1950: 214), la distinción es corrientemente sostenida en estudios sincrónicos, sobre todo en atención a una diferencia de tratamiento formal (v. e. g. Cabré Monné, 2010: 9). A esa diferencia y el correlativo reconocimiento nos referimos, en este trabajo, de modo particular.

3 Por el carácter representativo de la ciudad en el marco de dicha área, véase e. g. Fontanella de Weinberg y otros (1991: 36). 
La disparidad verificada comparativamente en ambos cuerpos de datos, que ratifica la caracterización que ensayamos para ambos tipos de intervención, constituye el aporte principal de este artículo.

\section{Acerca de la hipercaracterización grafémica}

El fenómeno que procuramos contribuir a deslindar y analizar que denominaremos hipercaracterización grafémica ha sido indicado como posible recorte objetual de importancia para el análisis sociolingüístico por Gómez Capuz y Rodríguez González (2002: 269-270), quienes afirman que "[u]n aspecto todavía poco estudiado de la ortografía moderna es lo que Pratt (1973) denomina «hipercaracterización ortográfica», que tiene lugar cuando una palabra es ortografiada en español de forma no normativa, pero no por desconocimiento de la norma sino para expresar un significado adicional" (énfasis nuestro).

La escritura deliberadamente no normativa de palabras españolas que nos interesa asienta su peculiaridad en el uso de recursos escriturarios de otras lenguas, por lo que resulta analíticamente accesible desde dos perspectivas diferentes, en relación con las cuales se presenta como intersección:

(a) la indagación sobre el modo en que se imbrican en producciones únicas recursos de más de una lengua, con limitación en este caso al nivel grafémico (lo cual involucra de manera privilegiada las correspondencias grafémico-fonológicas), y

(b) el análisis de la manipulación formal (Winter-Froemel, 2016: 20) supuesta por el juego lingüístico ${ }^{4}$, que puede abrevar en más de una lengua para conseguir sus efectos.

Las ocurrencias que nos interesan en particular, así, son las de una escritura de palabras españolas que resulta inesperada o marcada respecto de las convenciones escriturarias del español por implicar la recurrencia a convenciones exógenas. En idéntico sentido, en su conocido estudio sobre los rótulos publicitarios en Tenerife con énfasis en el influjo del inglés, Medina López (1991: 126) habla de "hipercorrección ortográfica” como del fenómeno que consiste en

4 Preferimos hablar de juego lingüístico y no de juego de palabras - aunque se ha defendido reiteradamente el valor hiperonímico de este último sintagma técnico, cuando menos en producciones en lengua inglesa, sobre la base de la difusión de su uso (v. e. g. Winter-Froemel, 2016: 37)- por cuanto enfocamos manipulaciones formales operadas sobre palabras, pero a nivel subléxico. Formas como las que identificamos han sido consideradas instancias de "juego de palabras en sentido amplio", en la medida en que se observa en ellas una "modificación ortográfica de ítems léxicos sin involucrar significados diferentes" en términos referenciales, en el reciente estudio de Thaler (2016: 59, traducción nuestra). 
"altera[r] la grafía castellana para que adquiera características parecidas a la inglesa", y ejemplifica con denominaciones comerciales como Deko Albert Canarias, Impakto 88 o Canarifot ${ }^{5}$.

Sobre la base de lo expuesto, iniciamos nuestra discusión con la de las siguientes ocurrencias registradas en el ámbito comercial de Bahía Blanca6:

(1) (Inscripción en el volante publicitario de una concesionaria de automóviles, referida a las ofertas de la marca Volkswagen:)

El auto korrekto

(2) (Denominación de una tienda de ropa que comercializa prendas de talles especiales:) ACkTITUD

(3) (Denominación de una tienda de marroquinería, bijouterie y accesorios:) Wapas

Según puede verse, en (1) se ha sustituido $\langle c\rangle$ por $\langle k\rangle$ y en (2) se ha hecho corresponder / k/ con el dígrafo 〈ck〉, en ambos casos enfrentando las convenciones españolas. El uso de 〈k〉 para /k/ española ha sido descripto reiteradamente en otros contextos, en particular en vinculación con la actual comunicación tecnológicamente mediada, en la que, como lo han señalado Sarmiento y Vilches (2009: 3), cuando se usa 〈k〉 no canónicamente es en general para sustituir al dígrafo 〈qu〉, i. e., en la procuración de un texto más breve en lo que respecta al número de caracteres, lo cual reviste importancia en dispositivos que requieran de esa economía. En el caso que nos ocupa, y en ausencia de tal requerimiento, el propósito de la recurrencia a 〈k〉 pasa primariamente por no escribir “como todos", i. e., por llamar la atención, y de modo más específico por generar en el otro una inferencia de manera (Winter-Froemel y Onysko, 2012) respecto de la elección divergente’. Esa

5 A pesar de esta coincidencia, señalamos igualmente nuestra preferencia - a la que nos ceñiremos en el artículo- (a) por hipercaracterización sobre hipercorrección en este caso, dado que consideramos útil la especialización simultánea de ambos términos en relación con la diferencia que trazamos (v. infra), y (b) por grafémica sobre ortográfica, por cuanto el prefijo de este último adjetivo remite a una corrección normativa que, precisamente, resulta eludida en las formas enfocadas.

6 Reproducimos con fidelidad, en todos los ejemplos, las configuraciones grafémicas registradas. Esa fidelidad abarca la combinación de mayúsculas y minúsculas del original. Solamente es nuestro el destacado en itálicas de los recursos grafémicos que nos interesan, con la única excepción del ejemplo (2), en el que <k> aparece destacada en el original por el uso simultáneo de itálica y minúscula.

7 Aun cuando se requiera una exploración más ajustada para precisar su aplicabilidad estricta, trasladamos aquí las consideraciones que Winter-Froemel y Onysko realizan sobre piezas léxicas, cuya importación es esperable (o no) según haya (o no) "equivalentes semánticos” en la lengua recipiente, a configuraciones formales esperables (o no) de acuerdo con la fijación de las normas respectivas (en este caso, escriturarias). 
recurrencia a 〈k〉 puede considerarse interlingüística en la medida en que "no es grafía tradicional en español” (Chacón Berruga, 2012 [2001]: 107): incluso en la nueva Ortografía de la lengua española (Real Academia Española, 2010: § 6.2.2.4.2.1), el uso del grafema es referido siempre en relación con la escritura de palabras provenientes de otras lenguas ${ }^{8}$. A pesar de ello, "la tendencia a emplear la consonante k, poco utilizada en el español escrito estándar", ha sido descripta igualmente como "muy generalizada en los textos electrónicos, como marca de este sociolecto y, más generalmente, del lenguaje juvenil” (Vela Delfa, 2006: 690-691). Aunque en este último caso se destaca igualmente su valor "reductor" (para la graficación de /k/ ante vocal anterior, o para la escritura "sintética" de la secuencia/ka/), lo expuesto puede sintetizarse como una expansión del uso de 〈k〉, que desborda su ubicación periférica en el sistema grafémico español con limitación funcional a la escritura de palabras exógenas sin adaptación (completa). Su empleo no característico fuera del "estilo electrónico" puede sincrónicamente asociarse, así, tanto a la informalidad vinculada con la juventud como a la presunta extranjeridad que ejemplifica la ocurrencia citada, y de la estabilidad de esta última asociación puede considerarse prueba la reiteración de las ocurrencias en las que 〈k〉 se agrega a la esperable <c> para la graficación de /k/ española, como en ACkTITUD o en Qubicko (v. infra).

La misma limitación regular a la escritura de palabras exógenas sin adaptación (completa) le cabe al grafema 〈w〉, ingresado al español "por la vía del préstamo" y "emplea[do] en voces procedentes de otras lenguas" (Giménez Folqués, 2012: 68), cuyo uso "expresivo" y consiguiente expansión se constata mucho menos frecuentemente que el de 〈k〉. Implica este grafema el ejemplo (3), en el que se ha partido del reconocimiento de la alternancia oral entre ['gwapa] y ['wapa] y, en la representación grafémica de este último alternante, se ha empleado $\langle w\rangle$ en lugar de $\langle u\rangle$.

En la bibliografía dedicada a estas cuestiones, la sustitución de $\langle k\rangle$ por $\langle c\rangle$ ha sido entendida reiteradamente como una instancia de extranjerización, como lo hacen Gómez Capuz y Rodríguez González (2002: 270) al clasificarla como “hipercaracterización ortográfica” sobre el modelo provisto principalmente por el inglés. Los mismos autores (ibid., n. 5) se refieren a escrituras con “hipercaracterización ortográfica debida a influencia del inglés” también en el caso de escrituras no normativas de palabras españolas que extienden el uso de 〈w〉.

Los ejemplos comentados, en los que recursos grafémicos inesperados en la escritura de palabras españolas sustituyen a los regulares a tal fin, muestran con claridad la diferencia

8 Aunque en (1) la lengua invocada es el alemán, el uso de <k> para la escritura no normativa de palabras españolas ha sido "denunciado" reiteradamente como transferencia del inglés, como -con virulencia purista - lo hace Manrique (2007: 232): "La invasión del inglés, junto al mal conocimiento y uso que de esta lengua se hace en España, ha propiciado esta chapucería [...]. Se cree que se es más importante por escribir Pako, pelukeria, karniceria, koko, etc.". 
entre las configuraciones no normativas que nos interesan y otras posibles (en particular, los errores ortográficos). No se trata solamente de escribir diferente una palabra dada, sino

(a) de escribirla de un modo en el que no se escribiría de limitarse el productor a las correspondencias grafémico-fonológicas regulares (no solo por ser las normativas, sino sobre todo por ser las más extendidas en el uso) del español, y

(b) de escribirla diferente para "decir" algo diferente de lo que "dice” su configuración grafémica convencionalizada.

De esta manera, el carácter exógeno de algunos de los recursos empleados en los que Medina López (1991) denomina "rótulos publicitarios" no es extraño a su selección, por cuanto el valor connotativo que la extranjeridad invoca es expresamente procurado.

En lo que atañe a la configuración formal que resulta de la combinación en configuraciones únicas de recursos convencionales españoles y de recursos exógenos, en el área dialectal sobre la que trabajamos observamos también grados mayores de refinamiento en el juego lingüístico que nos interesa, enancados en la posibilidad de una "doble lectura", según se siga la pauta de lectura española o se recuperen correspondencias grafémico-fonológicas no españolas, miméticas respecto de un modelo exógeno, que se hallan extendidas en el tratamiento oral local de importaciones léxicas (v. e. g. Hipperdinger, 2016). Ilustra esa posibilidad la denominación de un "almacén de diseño" de Bahía Blanca, escrita como sigue:

\section{(4) LooKaaS}

En este caso, la lectura española resulta en /'lokas/, pero es igualmente posible una lectura alternativa (que hemos corroborado en la realización oral del nombre del emprendimiento por parte de hablantes bahienses) como /'luk as/, que recupera la correspondencia de 〈0o〉 con / $/{ }^{9}$, extendida para la realización oral de numerosas importaciones léxicas del inglés en la variedad territorial de español de la que nos ocupamos. El juego lingüístico, en casos como este, consiste en mezclar recursos de dos lenguas para habilitar interpretaciones alternativas según se “lea en español” o no (para LooKaaS, así, 'locas’ y ‘míranos’).

Otras dos fuentes principales de generación de variantes encontramos en este juego con la grafía que intentamos poner en foco: la que implica elementos diacríticos y la que opera sobre el límite entre las palabras. En el primer caso, una marca gráfica con valor diacrítico en una lengua es trasladada a una palabra en otra lengua, en el marco de cuyas convenciones escriturarias el referido valor resulta anulado y es, subsiguientemente, reinterpretado. En el

9 No se verifica una correspondencia no española equiparable que implique 〈aa〉. 
segundo caso, a la manipulación extranjerizadora de la grafía se agrega la alteración de la demarcación convencional entre palabras en la cadena escrita, por supresión del blanco tipográfico. Las siguientes denominaciones comerciales de Bahía Blanca constituyen ejemplos:

(5) Antigüedades

Estilo's

(6) Kebesha

y mas... ropa deportiva ${ }^{10}$

A diferencia de otros sintagmas atestiguados en denominaciones comerciales de la ciudad en los que el signo gráfico 〈’ seguido de 〈s〉 se ajusta a las convenciones escriturarias del inglés, a nivel grafémico-morfológico por tratarse del llamado "genitivo sajón”, se trata en (5) de la extranjerización de una palabra española flexionada en plural: la representación convencional 〈's〉, que en inglés sirve como marca de posesión, pasa a servir aquí para hacer "Ilamativa” la escritura del plural españoli1. Un ejemplo que manifiesta la diferencia señalada es el de la denominación de la peluquería canina Bahia’s dogs, en la que el recurso aparece con su valor original y que muestra adicionalmente la omisión de la tilde española, en apariencia para anglicizar la configuración grafémica del topónimo. Si bien esta última omisión puede ser considerada también una instancia de manipulación interlingüística, es de notar que no podría identificársela como tal fuera de esa concurrencia, dada la frecuencia con que se registra la omisión de la tilde normativa en el marco de errores ortográficos ${ }^{12}$.

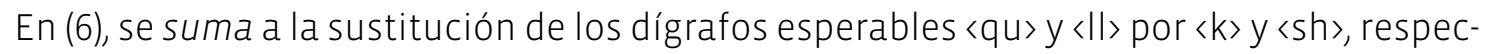
tivamente, la supresión del blanco tipográfico entre el pronombre exclamativo y el adjetivo. Esta última supresión solo merece destacarse en el marco del juego interlingüístico que

10 Por el dígrafo 〈sh〉, véase por ejemplo Giménez Folqués (2012: 66ss.).

11 También se refiere al “uso del genitivo sajón” Medina López en su trabajo ya citado sobre los rótulos publicitarios en español canario, y lo separa de la "hipercorrección ortográfica” por su carácter de transferencia morfológica (1991: 127); no obstante, y si bien casi todos sus ejemplos ilustran el caso del genitivo propiamente dicho (Pepe's cerámica, Alejandro's), algunos pueden considerarse también casos de escritura "Ilamativa” del plural (Novia's, Multicines Oscar's). Por su parte, Silva-Corvalán y Enrique-Arias (2017: 313) relevan asimismo "la adopción del morfema genitivo del inglés" (con limitación estricta a su carácter de transferencia morfológica) en el ámbito específico de que nos ocupamos, registrándolo en España "en los nombres de ciertos locales comerciales (especialmente bares estilo pub inglés), que aparecen escritos así: Paco's, Pepe's, etc.".

12 Así, y aunque la omisión de la tilde normativa no tiene la visibilidad de la supresión de blancos tipográficos a la que nos referimos ni es incuestionable en todos los casos su carácter deliberado, incluso cuando es posible suponérselo le cabe el mismo comentario que sobre esta última realizamos infra, en el sentido de que se limita a agregar "rareza" a una forma ya extranjerizada. 
intentamos circunscribir en la medida en que constituye un indicador más de la intención rupturista del productor: contribuye a "enrarecer" la forma manipulada, pero no se asocia per se a otra lengua.

En consecuencia (y síntesis), la hipercaracterización grafémica extranjerizadora que nos interesa pasa siempre por una sustitución (la de un recurso convencional en español por uno convencional en, y asociado con, otra lengua). A su vez, esa sustitución puede, en términos del número de elementos implicados, clasificarse del siguiente modo:

(a) amplificación (cuando se emplean más elementos en la configuración grafémica de que se trata que en la española regular), como en los casos de ACkTITUD y Estilo's;

(b) reducción (cuando se emplean menos), como en el caso de Kebesha;

(c) sustitución propiamente dicha (cuando no hay amplificación ni reducción en el sentido descripto), como en el caso de korrekto.

\section{Hipercaracterización en letra pequeña: revisando la Guía Clasificada}

Sobre la base de lo que llevamos dicho, consignamos seguidamente los resultados de nuestro registro de configuraciones grafémicas extranjerizadas en la sección Guía Clasificada de la Guía Telefónica de Bahía Blanca 2016 (Telefónica - Páginas Amarillas)². Esa Guía Clasificada contiene los números de teléfono -incluidos o no en avisos publicitarios- de emprendimientos industriales, comerciales y de servicios, cuyas denominaciones nos interesan específicamente ${ }^{14}$. De acuerdo con lo expuesto supra, clasificamos las denominaciones que registramos en formas en las que se ha operado amplificación, reducción o (mera) sustitución. Cuando en la configuración grafémica de una misma denominación comercial se han operado intervenciones diferentes según esta clasificación, consignamos la forma de que se trata

13 Puede verse un especialmente interesante estudio desarrollado sobre páginas amarillas de directorios telefónicos en la revisión diacrónica ofrecida para Helsinki por Pahta y Taavitsainen, quienes documentan el progresivo aumento de la presencia del inglés en el marco de lo que describen como tendencia global "in commercial advertising and naming practices" (2004: 168) y que, como se verá, nuestro estudio atestigua también.

14 Excluimos de nuestro registro las denominaciones comerciales que implican configuraciones grafémicas jurídicamente fijadas con antelación a su elección para el fin de referencia, entre las cuales predominan ampliamente los apellidos no hispánicos. De acuerdo con esto último, por ejemplo, no consideramos casos como el de la denominación comercial Buena Maizon, que (en el marco también de un juego interlingüístico) involucra el apellido de la familia fundadora de la empresa metalúrgica homónima, Maizon. En cambio, incluimos los apodos intervenidos del modo que nos interesa que encontramos como denominaciones comerciales en la Guía. 
de modo repetido bajo los rótulos que corresponden, y señalamos con itálicas la instancia específica de manipulación a la que en cada caso hacemos referencia ${ }^{15}$.

(a) Hipercaracterización por amplificación:

Carma's ${ }^{16}$
Chino's
Diva's
Elegante's
Guappa's
Kala's
Kesote's
Kokass
Krokantito's
Libélula's
Nono's
Petty
Pilcha's
Qubicko

15 El orden en que enlistamos las formas sigue un criterio alfabético. Algunas de estas denominaciones aparecen una única vez en la Guía, mientras que otras lo hacen reiteradamente. Así, por ejemplo, el nombre de la tienda Elegante's solamente aparece en la página 55, mientras que el nombre del emprendimiento en tecnología en construcciones Qubicko aparece en las páginas 29, 63-64, 74, 82,161 y 171 . Dado que esa diferencia no es relevante para nuestros fines inmediatos, omitimos las precisiones correspondientes. Otra clase de información a la que no haremos referencia aquí tiene que ver con el carácter (estrictamente local o no) de las distintas empresas, ya que no se liga al criterio que empleamos para el registro.

16 Incluimos también, como en este caso o el de nono, palabras que fueron oportunamente importadas pero que, por su amplia extensión de uso actual y su completa adaptación al español, no resultan reconocibles como exógenas para el conjunto de los hablantes.

17 De acuerdo con lo que llevamos dicho, no incluimos aquí casos en los que la interpretación de 〈's〉 como plural no es posible, como en el nombre de la empresa de venta de productos de acuario Daniel's Mar. Persiste, no obstante, el problema de que algunas de las denominaciones que incluyen 〈's〉 que constatamos pueden implicar o bien el genitivo en su uso original o bien su reinterpretación como plural. En la Guía, es este particularmente el caso de Pato's y Perla’s (siendo Pato un apodo de uso extendido y Perla un nombre de mujer), que — por lo mismo- excluimos de la lista.

18 No incluimos otras dos formas registradas similares a Qubicko, Kubic y Qbik, por tratarse de intervenciones sobre una base importada del inglés (cubic). No obstante, nos interesa dejar constancia de la diferencia en el uso de 〈q〉 entre Qubicko y Qbik, ya que en este último caso la intervención ("reductora") consiste en atribuir al grafema el valor fónico de su nombre (/ku/), lo cual, aunque no en la Guía, se constata en el ámbito comercial de la ciudad en relación también con otros grafemas, como lo muestra la denominación del local de servicios para mascotas D’Pelos. Esta 
Que pollo's

Rikissimos ${ }^{19}$

Riko's

Taco's

(b) Hipercaracterización por reducción:

Fainalind

Kesote's

Kika

Kike

Kito

Niki

Polienvas

Rikissimos

Stylo Urbano 20

(c) Hipercaracterización por (mera) sustitución:

Kactus

Kala's

Karla S

Kika

Kokass

Krokantito's

última denominación es interesante porque muestra, adicionalmente, un uso del apóstrofe no ligado ya al genitivo sajón (que es el único que se encuentra en la Guía): en D’Pelos el apóstrofe marca, del modo corriente, una elisión — que a nivel grafémico efectivamente se ha operado-, siendo condición para la lectura la referida adjudicación del valor fónico del nombre del grafema al grafema mismo. Este uso del apóstrofe y el que aparece en la escritura intervenida del plural que registramos pueden considerarse, en la terminología de Pires (2000), casos de apóstrofe elisivo y no elisivo, respectivamente.

19 Nótese que en todos los casos en que se reitera un grafema manteniendo su valor fónico no se pasa de una duplicación. Entendemos que justamente de esto depende que pueda pensarse en el modelo de otra lengua, ya que la reiteración de un mismo grafema es un rasgo expresivo no interlingüístico muy comúnmente registrado en otros contextos, en especial en la escritura tecnológicamente mediada, pero típicamente con una repetición mayor (v. e. g. Cutler, 2016: 825s., y para el caso del español bonaerense Cantamutto, 2014: 76).

20 El uso de $\langle y\rangle$ por $\langle i\rangle$ ha sido registrado reiteradamente, e identificado como influjo del inglés. Así lo hace por ejemplo Rodríguez Medina (2014: 410), rotulándolo como "hipercaracterización ortográfica”, en su estudio sobre el anglicismo en gimnasios españoles. En posición final, Rodríguez González (2013: 134) considera asimismo sus ocurrencias casos de "hipercaracterizacion gráfica”. 


Lo de Naty
Mapy
Mity
Petty
Qubicko
Riko's
Stylo Urbano
Sygnos
Zentriko21
Zokalo

Como puede verse, los modos de intervención relevados no son muchos. Se emplean amplificando solamente el apóstrofe seguido de la $\langle$ s que marca el plural y una digrafización de consonan-

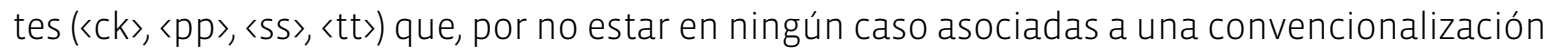
puntual mimética del tipo descripto supra, no tienen consecuencias sobre la realización oral. Un efecto de reducción en el número de grafemas tiene el uso de 〈k〉 por 〈qu〉 ya referido, y también lo tiene la elisión de vocales que se constata tanto en posición final postconsonántica (en Fainalind y Polienvas) como en posición inicial (en Stylo22). Por último, sin generar alteración en el número de grafemas se opera la sustitución de $\langle c\rangle$ por $\langle k\rangle$, así como por $\langle q\rangle$ ante $\langle u\rangle$, y la sustitución de 〈i por 〈y〉. Es de notar que la sustitución de <i〉 por 〈y〉 se liga estrechamente a la palabra de que se trata en Stylo y Sygnos, pero es de "aplicación mecánica” en posición final la que Rodríguez González (2013: 134) describe como la "característica terminación inglesa -y" que se emplea fuera de esa lengua "por un deseo de dar apariencia inglesa a algún morfema español o foráneo".

En consecuencia, los únicos modos de intervención que se reiteran son la reinterpretación del genitivo inglés, la digrafización (irrelevante en relación con la correspondencia oral) de consonantes, el uso de 〈k〉 por 〈qu〉 y por 〈c〉y, en posición final postconsonántica, la elisión de vocales (que configura apócopes irregulares para el español) y la sustitución de 〈i〉 por 〈y〉. Incluso excluyendo el uso de $\langle k\rangle$ de entre las hipercaracterizaciones extranjerizadoras por su referida difusión, dado que, como hemos dicho, puede argumentarse - con independencia de su origen- una motivación "intrasistemática” para la extensión de su uso, no puede atribuir-

21 Sin desestimar la posibilidad de que la forma haya sido modelada sobre Zentrum, del alemán, parece más probable que la sustitución de 〈c〉 por 〈z〉 se trate de un juego no interlingüístico, montado sobre una constatación de las posibilidades de homofonía entre formas heterógrafas que ofrece la variedad regional de español de que se trata (por ejemplo, y aunque fuera de la Guía, hemos constatado en tal sentido Colores z $Z^{Z}$ ubidos o Zel-este).

22 Esta misma configuración ha sido registrada por Rodríguez Medina (2014: 410), quien la describe como un "híbrido entre el inglés style y el español estilo, en el que se opta por una grafía próxima a la inglesa”. 
se a la casualidad que la única otra lengua en la que puede haberse abrevado para aumentar las posibilidades escriturarias del español con fines lúdicos que, simultáneamente, puede haber sido el modelo para todas estas intervenciones sea justamente el inglés, la lengua que Calvet (1999) ha llamado hipercentral. Como lo señalan Gómez Capuz y Rodríguez González (2002: 270), “Pratt ([hace más de cuatro décadas, en] 1973) ya insistía en que la mayoría de las hipercaracterizaciones ortográficas del español actual se debían a la influencia de este idioma”.

\section{Configuraciones grafémicas hipercorrectas de importaciones léxicas}

El fenómeno del que nos venimos ocupando ha sido a veces englobado sin diferenciación junto con otro, con el que sin duda está estrechamente vinculado pero del que no solo es distinguible, sino que merece ser distinguido: el de la intervención sobre configuraciones escritas de palabras identificadas como importaciones. La indiferenciación referida puede apreciarse en (y quizás hasta haya sido propiciada por) la difundida definición ofrecida por Pratt, en su contribución pionera ya citada, cuando dice: "[E]ntiendo por «hipercaracterización ortográfica» el fenómeno de alterar la grafía de una forma para que llegue a ostentar características del sistema ortográfico de otra lengua" (1973: 63).

Obviamente la "forma” de la que se trata, salvo que se establezca una precisión al respecto, puede ser o bien la de una palabra reconocida como patrimonial, o bien la de una palabra reconocida como exógena. Específicamente, la distinción que enfocamos se vincula con distintas acepciones del verbo ostentar: 'mostrar' y 'hacer gala de' (v. Real Academia Española, 2014). De esta manera,

(a) si la forma de palabra cuya escritura se altera se reconoce como española, la recurrencia a características grafémicas de otra lengua ‘muestra'('indica’) la intención de jugar, mientras que

(b) si la forma de palabra cuya escritura se altera se reconoce como no española, los recursos grafémicos de otra lengua que resulten involucrados los emplea el productor para 'hacer gala de’ su conocimiento (o, mejor, no ignorancia) de las características escriturarias de la lengua donante.

En el caso de (a), una forma española resulta extranjerizada. En el caso de (b), una forma exógena resulta hiperextranjerizada ${ }^{23}$. La manipulación extranjerizadora de la grafía de palabras españolas es inherentemente lúdica, como ya lo hemos señalado. En cambio, el productor que hipercorrige su escritura de una palabra que reconoce como no española no necesariamente

23 Por la categoría de hyper-foreignism, véanse Hock y Joseph (1996: 270) y Winter-Froemel (2008: 165, 2011: 61 et alibi). 
se involucra en un juego lingüístico: de hecho, comúnmente los alternantes hipercorrectos verificados para las importaciones léxicas son el producto de intentos "serios" de acercamiento al target constituido por la configuración escrita convencionalizada en la lengua de origen.

Son ejemplos de configuraciones grafémicas sometidas a hipercorrección las siguientes denominaciones comerciales, registradas también en la sección Guía Clasificada de la Guía Telefónica de Bahía Blanca 201624:

\section{Buone Vita}

Fàcile

Le femme 25

Milanno

Así, y aunque por supuesto es posible que se operen intervenciones lúdicas sobre palabras reconocidas como exógenas (de lo que es ejemplo la denominación comercial Beautyfull), puede afirmarse, en relación con la distinción que nos interesa, que quien hipercaracteriza se esfuerza por escribir palabras españolas como sabe que no se escriben, mientras que quien hipercorrige palabras que reconoce como exógenas normalmente las escribe de ese modo porque cree que es así como se escriben en la lengua de origen.

En relación con este último modo de intervención, llevamos a cabo un relevamiento de los alternantes escriturarios hipercorrectos para importaciones léxicas en el mismo ámbito y ubicación lingüístico-territorial que el anteriormente informado. Efectuamos un registro de las configuraciones correspondientes en los avisadores y menúes de setenta emprendimientos comerciales del rubro gastronómico en la zona céntrica de la ciudad de Bahía Blanca, cuyo resultado es el siguiente ${ }^{26}$.

bagell (< bagel) $)^{27}$

24 Señalamos en cada caso con itálicas la hipercorrección operada. El orden enumerativo sigue, otra vez, un criterio alfabético.

25 La extensión del uso de le como determinante para sustantivos de origen francés, con neutralización de la diferencia de género gramatical, se aprecia en diversas denominaciones comerciales de la ciudad (Le coquette, Le baguette, etc.).

26 Como en las listas anteriores, no agregamos información irrelevante a nuestros fines actuales, seguimos un criterio alfabético y señalamos con itálicas la intervención que en cada caso nos interesa. En esta lista agregamos, además, las configuraciones grafémicas que constituyeron el target en cada caso.

27 Para esta especialidad de la cocina judía tomamos como forma de referencia la más difundida tanto en español como en otras lenguas, como el alemán y el inglés (por vía del cual parece haberse operado el ingreso del término al uso en nuestras latitudes). 


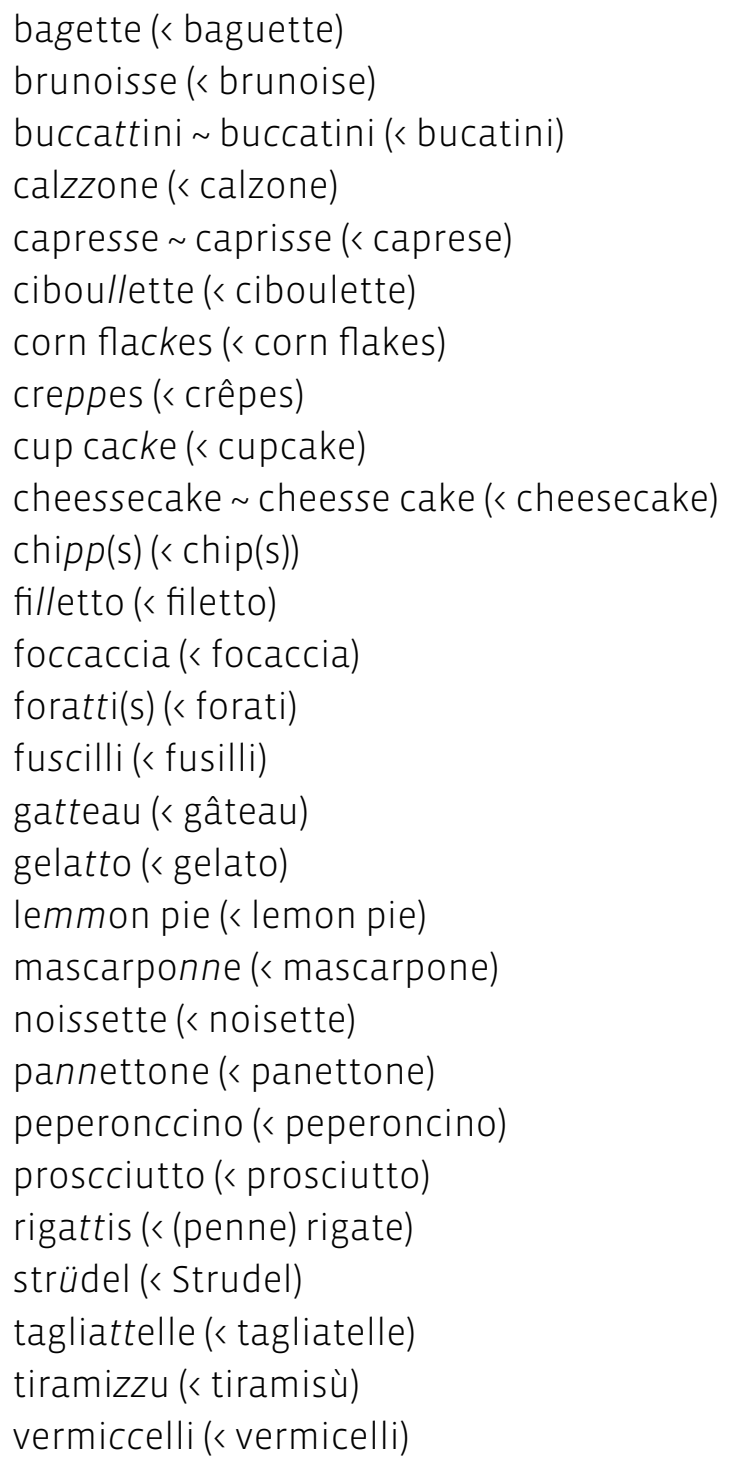

Según puede apreciarse, también en este caso las intervenciones pueden clasificarse en las que se limitan a sustituir un grafema por otro (como en strüdel, en que la 〈ü〉 característicamente alemana sustituyó una <u> que podría haber sido española), las que producen una reducción (como la supresión de <u> en bagette) y las que amplifican el número de grafemas (como en ciboullette). Sin embargo, las grafías hipercorrectas enlistadas se diferencian de las hipercaracterizadas a las que nos referimos antes por cuanto en este caso la "innovación" recupera siempre un recurso efectivamente presente en la respectiva lengua donante, aun cuando no aparezca en la escritura de esas lenguas no solo en la forma de palabra pertinente, sino tampoco en la posición de que se trata (lo que ejemplifica chipp(s)) o, incluso, no se ajuste a la correspondencia grafémico-fonológica invocada (como lo ilustra tiramizzu).

La prueba privilegiada de esa intención de ajustar la producción propia a las configura- 
ciones de origen es que recursos grafémicos identificados con solo una de las lenguas donantes aparecen, exclusivamente, en la hipercorrección de las configuraciones escritas de piezas léxicas de esa lengua (como lo transparentan calzzone, fuscilli y proscciutto/proscciutto para el caso del italiano o strüdel para el caso del alemán).

\section{Conclusiones}

Hemos planteado en este artículo la conveniencia de deslindar, con la mayor claridad posible, los dos fenómenos registrables en la escritura, vinculados pero diferentes, que llamamos de hipercaracterización e hipercorrección, entendiendo que, aunque en ambos casos se implique la recurrencia a convenciones exógenas por parte de miembros de una misma comunidad de habla, el primero implica una extranjerización y el segundo, una hiperextranjerización.

Para usos de español bonaerense, sobre datos obtenidos para una misma ciudad, verificamos una diferencia concomitante entre los dos fenómenos: en los alternantes hipercorrectos constatados en la escritura de importaciones léxicas se implica regularmente el uso de convenciones grafémicas de la que, en cada caso, es identificada como lengua de origen, mientras que las que se emplean en la hipercaracterización de palabras españolas, en cambio, remiten típicamente al inglés. Como sostiene Medina López (2004 [1998]: 29-30), “la hipercaracterización ortográfica, así como el uso del genitivo sajón, se muestran como dos mecanismos frecuentes en este tipo de rótulos [comerciales] y suponen una motivación económica y material que está unida a la aceptación general y de estatus que en determinados ambientes tiene el anglicismo para acuñar o modificar nombres españoles” (énfasis nuestro).

En relación con la hipercaracterización, en particular, sumamos con esta contribución evidencia de detalle al conocimiento del influjo derivado de la presencia ambiental de la lengua hipercentral calvetiana en el español bonaerense, en este caso en el marco de un juego lingüístico que, aunque parecería en principio abierto a una creatividad ilimitada, demuestra basarse —como todos los juegos - en un muy restringido conjunto de acuerdos: pudiendo elegir, solo se usa lo que se sabe que va a entenderse (y entenderse como manipulación, no como error). La confianza de los productores en esa comprensión (la de quienquiera que lea la denominación de un local comercial, in situ, en la Guía o en otras formas de publicidad) descansa privilegiadamente en la posición hipercentral contemporánea del inglés.

\section{Bibliografía citada}

Cabré Monné, Teresa, 2010: "La adaptación de préstamos en catalán”, conferencia presentada en el Ciclo de Conferencias 2010 "El espacio ibérico de las lenguas".

Calvet, Louis-Jean, 1999: Pour une écologie des langues du monde, Paris: Plon. 
Cantamutto, Lucía, 2014: "El discurso de los mensajes de texto en el habla adolescente del español bonaerense" en Alejandro Parinı y Mabel Giammatteo (eds.): Lenguaje, discurso e interacción en los espacios virtuales, Mendoza: Universidad Nacional de Cuyo-Sociedad Argentina de Lingüística, 65-81.

Chacón Berruga, Teudiselo, 2012 [2001]: Ortografía normativa del español (vol. I), quinta edición, Madrid: Universidad Nacional de Educación a Distancia.

Cutler, Cecilia, 2016: "'Ets jast ma bo000000000000': Social meanings of Scottish accents on YouTube" en Lauren Squires (ed.): English in Computer-Mediated Communication, Berlin: Walter de Gruyter, 69-98.

Fontanella de Weinberg, María Beatriz, 1987: El español bonaerense. Cuatro siglos de evolución lingüística (1580-1980), Buenos Aires: Hachette.

Fontanella de Weinberg, María Beatriz, 2000: “El español bonaerense” en María Beatriz Fontanella DE WeInBerg (coord.): El español de la Argentina y sus variedades regionales, Buenos Aires: Edicial, 37-61.

Fontanella de Weinberg, María Beatriz, y otros, 1991: Lengua e inmigración. Mantenimiento y cambio de lenguas inmigratorias, Bahía Blanca: Universidad Nacional del Sur

Giménez Folqués, David, 2012: Los extranjerismos en el español académico del siglo XXI, València: Universitat de València.

Gómez Capuz, Juan, y Félix Rodriguez González, 2002: "El lenguaje de los soldados" en Félix Rodríguez GonzAlez (coord.): El lenguaje de los jóvenes, Barcelona: Ariel, 265-290.

Haspelmath, Martin, 2009: "Lexical borrowing: Concepts and issues" en Martin Haspelmath y Uri TADMOR (eds.): Loanwords in the World's Languages. A Comparative Handbook, Berlin: Walter de Gruyter, 35-54.

Haugen, Einar, 1950: “The analysis of linguistic borrowing”, Language 26, 210-231.

HipPeRdinger, Yolanda, 2016: "La mímesis (y sus límites) en la realización oral de importaciones léxicas” en Ubiratã Kickhöfel Alves (org.): Aquisição fonético-fonológica de língua estrangeira, Campinas: Pontes Editores, 255-265.

Hock, Hans, y Brian Joseph, 1996: Language History, Language Change, and Language Relationship, Berlin / New York: Mouton de Gruyter.

ManriQue, Miguel, 2007: El falso progresismo, Madrid: Visión Net.

Medina López, Javier, 1991: “Los anglicismos. A propósito de los rótulos publicitarios”, Lexis XV (1), 119-128. 
Medina López, Javier, 2004 [1998]: El anglicismo en el español actual, segunda edición, Madrid: Arco Libros.

Pahta, Päivi, e Irma TaAvitsainen, 2004: "Creating Images through English on Yellow Pages: Multilingual Practices in Advertising in the Helsinki Region", Nordic Journal of English Studies 3 (2), 167-185.

PIRES, Mat, 2000 : "Leçons de Gram'hair: fonctions de l'apostrophe en onomastique commerciale”, Langage et société 91, 59-86.

Pratt, Chris, 1973: "El lenguaje de los medios de comunicación de masas: algunos aspectos", Filología Moderna 46-47, 63-87.

Real Academia Española, 2010: Ortografía de la lengua española, Madrid: Espasa.

Real Academia Española, 2014: Diccionario de la lengua española, vigesimotercera edición [disponible en http://www.rae.es/obras-academicas/diccionarios/diccionario-de-la-lengua-espanola, fecha de consulta: 20 de octubre de 2017].

Rodriguez Gonzalez, Félix, 2013: “Pseudoanglicismos en español actual. Revisión crítica y tratamiento lexicográfico”, Revista Española de Lingüística 43 (1), 123-168.

Rodriguez Medina, María Jesús, 2014: "Anglicismos en el léxico de las actividades deportivas de los gimnasios españoles", Lexis XXXVIII (2), 401-427.

SARmiento, Ramón, y Fernando Vilches, 2009: “La calidad de la lengua en la red” en Ramón SaRmiento y Fernando Vilches (coords.): La calidad del español en la red. Nuevos usos de la lengua en los medios digitales, Barcelona: Ariel, 1-7.

Silva-Corvalán, Carmen, y Andrés Enrique-Arias, 2017 [2001]: Sociolingüística y pragmática del español, segunda edición, Washington, DC: Georgetown University Press.

ThaleR, Verena, 2016: "Varieties of Wordplay" en Sebastian KnosPe, Alexander Onysko y Maik Goth (eds.): Crossing Languages to Play with Words. Multidisciplinary Perspectives, Berlin: Walter de Gruyter, 47-62.

Vela Delfa, Cristina, 2006: El correo electrónico: el nacimiento de un nuevo género. Tesis de doctorado, Universidad Complutense de Madrid.

Winter-Froemel, Esme, 2008: "Studying loanwords and loanword integration: Two criteria of conformity", Newcastle Working Papers in Linguistics 14, 156-176.

Winter-Froemel, Esme, 2011: Entlehnung in der Kommunikation und im Sprachwandel. Theorie und Analysen zum Französischen, Berlin: Walter de Gruyter. 
Winter-Froemel, Esme, 2016: "Approaching Wordplay" en Sebastian Knospe, Alexander Onysko y Maik Goтн (eds.): Crossing Languages to Play with Words. Multidisciplinary Perspectives, Berlin: Walter de Gruyter, 11-46.

Winter-Froemel, Esme, y Alexander Onysko, 2012: "Proposing a pragmatic distinction for lexical Anglicisms” en Cristiano Furiassi, Virginia Pulcini y Félix Rodríguez-González (eds.): The Anglicization of European Lexis, Amsterdam/Philadelphia: John Benjamins, 43-64. 